\title{
Entropy and Its Variational Principle for Locally Compact Metrizable Systems
}

\author{
André Caldas* $\quad$ Mauro Patrão ${ }^{\dagger}$
}

March 25, 2021

\begin{abstract}
For a given topological dynamical system $T: X \rightarrow X$ over a compact set $X$ with a metric $d$, the variational principle states that

$$
\sup _{\mu} h_{\mu}(T)=h(T)=h_{d}(T),
$$

where $h_{\mu}(T)$ is the Kolmogorov-Sinai entropy, with the supremum taken over every $T$-invariant probability measure, $h_{d}(T)$ is the Bowen entropy, and $h(T)$ is the topological entropy as defined by Adler, Konheim and McAndrew. In 9], the concept of topological entropy was adapted for the case where $T$ is a proper map and $X$ is locally compact separable and metrizable, and the variational principle was extended to

$$
\sup _{\mu} h_{\mu}(T)=h(T)=\min _{d} h_{d}(T),
$$

where the minimum is taken over every distance compatible with the topology of $X$. In the present work, we dropped the properness assumption, extending the above result for any continuous map $T$.

We also apply our results to extend some previous formulas for the topological entropy of continuous endomorphisms of connected Lie groups proved in [4. In particular, we prove that any linear transformation $T: V \rightarrow V$ over a finite dimensional vector space $V$ has null topological entropy.
\end{abstract}

\section{Introduction}

In this paper, we extend to continuous maps $T: X \rightarrow X$ defined over a metrizable locally compact separable space $X$ the variational principle for entropies. We have adapted the classical proof of variational principle due to Misiurewicz (see Theorem 8.6 of [11).

The original topological entropy was defined by Adler, Konheim and McAndrew (see [1]), by adapting the measure theoretic definition, which we call Kolmogorov-Sinai entropy. In this work, this will be called the $A K M$

\footnotetext{
* Departamento de Matemática - Universidade de Brasília-DF, Brasil. Supported by CNPq grant no. 140888/11-0.

${ }^{\dagger}$ Departamento de Matemática - Universidade de Brasília-DF, Brasil. Supported by CNPq grant no. 310790/09-3.
} 
entropy. By assuming the underlying space is equipped with a distance function, Bowen and Dinaburg (see [3] and [5]) have defined a different concept of entropy, which we shall call Bowen entropy. In the case of a compact metrizable space, this concept of entropy coincides with the AKM entropy. In particular, in the compact case, the value of the Bowen entropy does not depend on the particularly chosen distance. That is, for a given topological dynamical system $T: X \rightarrow X$ over a compact metric space $(X, d)$, Dinaburg and Bowen showed that

$$
\sup _{\mu} h_{\mu}(T)=h(T)=h_{d}(T)
$$

where the supremum is taken over every $T$-invariant probability measure. Probably for this reason, Bowen himself also named his entropy "topological entropy". However, for non compact spaces, the Bowen concept of entropy gives different values for equivalent distances over the same space, while the $A K M$ entropy would always be infinite. Later, Patrão realized that, even though the value of the Bowen entropy depends on the particular chosen distance function, by adapting the AKM entropy, to what we are calling topological entropy in this paper, a variational principle relating the measure theoretic Kolmogorov-Sinai entropy, the topological entropy and the Bowen entropy could be demonstrated, as long as the dynamical system $T$ could be extended to a metrizable one point compactification of $X$ (see [9]).

Inspired by the work of Adler, Konheim and McAndrew in 1 and the work of Patrão in [9], we extend the definition of topological entropy for arbitrary topological spaces. Inspired by the work of Bowen in 3, we simplify his definition of entropy $h_{d}(T)$ for non compact sets. We call it $d$-entropy, and denote it with superscript: $h^{d}(T)$. Then we show for the case where $X$ is locally compact metrizable and separable, that

$$
\sup _{\mu} h_{\mu}(T)=h(T)=\min _{d} h_{d}(T)=\min _{d} h^{d}(T),
$$

where the minimum is attained for every metric $d$ that can be extended to the one point compactification of $X$ (see Theorem 3.1). Notice that while the metric $d$ might come from the one point compactification, this does not mean that the dynamical system $T$ itself needs to have an extension to the one point compactification. In fact, $T$ can be any continuous system over a locally compact separable metrizable space $X$. This is a substantial improvement compared to Patrão's preview result in [9. In achieving such a result, it was fundamental that we were able to restate the different definitions of entropy using a unified approach that allowed easier comparisons between them.

We also apply our results to extend some previous formulas for the topological entropy of continuous endomorphisms of connected Lie groups proved in 4. In the case of a connected semisimple Lie group, without assuming the endomorphism to be surjective, we prove that its topological entropy always vanishes. In the case of a connected compact Lie group, a linear connected reductive Lie group or a connected nilpotent Lie group, without assuming the endomorphism to be surjective, we prove that the topological entropy coincides with the topological entropy of the endomorphism's restriction to the maximal connected and compact subgroup of the center. In particular, Proposition 4.6 implies that any linear transformation $T: V \rightarrow V$ over a finite 
dimensional vector space $V$ has null topological entropy. This extends the result of Proposition 4.2 in 9 .

The paper is organized in the following way. In Section 2, we recall some elementary definitions related to the different types of entropies, extend those definitions to a broader class of dynamical systems, and prove some fundamental facts which are used in the sequel. In Section 3, we prove our main result. And in Section 4, we apply the main result in order to determine the topological entropy for endomorphisms of some classes of Lie groups.

\section{Preliminaries}

This section is devoted to recalling some elementary definitions related to different types of entropy, and to proving some fundamental facts which are used in the sequel. We also extend the topological entropy - originally defined for compact systems - to an arbitrary topological dynamical system.

A topological dynamical system - or simply a dynamical system $T: X \rightarrow X$ is a continuous map $T$ defined over a topological space $X$. A measurable dynamical system $T: X \rightarrow X$ is a measurable map $T$ defined over a measurable space $X$. If we embed $X$ with the Borel $\sigma$-álgebra, a topological dynamical system becomes also a measurable dynamical system.

Recall that a family $\mathscr{A}$ of subsets of $X$ is a cover of $X$, or simply a cover, when

$$
X=\bigcup_{A \in \mathscr{A}} A .
$$

If the sets in $\mathscr{A}$ are disjoint, then we say that $\mathscr{A}$ is a partition of $X$. A subcover of $\mathscr{A}$ is a family $\mathscr{B} \subset \mathscr{A}$ which is itself a cover of $X$. If $Y \subset X$ and $\mathscr{A}$ is a cover of $X$, then we denote by $Y \cap \mathscr{A}$ the cover of $Y$ given by

$$
Y \cap \mathscr{A}=\{A \cap Y \mid A \in \mathscr{A}\} .
$$

We denote by $N(\mathscr{A})$ the least cardinality amongst the subcovers of $\mathscr{A}$. For $Y \subset X, N_{Y}(\mathscr{A})$ is a shorthand for $N(Y \cap \mathscr{A})$.

Given two covers $\mathscr{A}$ and $\mathscr{B}$ of an arbitrary set $X$, we say that $\mathscr{A}$ is finer then $\mathscr{B}$ or that $\mathscr{A}$ refines $\mathscr{B}$ - and write $\mathscr{B} \prec \mathscr{A}$ - when every element of $\mathscr{A}$ is a subset of some element of $\mathscr{B}$. We also say that $\mathscr{B}$ is coarser then $\mathscr{A}$. The relation $\prec$ is a preorder, and if we identify the symmetric covers (i.e.: covers $\mathscr{A}$ and $\mathscr{B}$ such that $\mathscr{A} \prec \mathscr{B}$ and $\mathscr{B} \prec \mathscr{A}$ ), we have a lattice. As usual, $\mathscr{A} \vee \mathscr{B}$ denotes the representative of the coarsest covers of $X$ that refines both $\mathscr{A}$ and $\mathscr{B}$, given by

$$
\mathscr{A} \vee \mathscr{B}=\{A \cap B \mid A \in \mathscr{A}, B \in \mathscr{B}, A \cap B \neq \emptyset\}
$$

Given a dynamical system $T: X \rightarrow X$ and a cover $\mathscr{A}$, for each $n \in \mathbb{N}$ we define

$$
\mathscr{A}^{n}=\mathscr{A} \vee T^{-1}(\mathscr{A}) \vee \cdots \vee T^{-(n-1)}(\mathscr{A})
$$

If we want to emphasise the dynamical system $T$, we write $\mathscr{A}_{T}^{n}$ instead. 


\subsection{Compactification}

The variational principle has been demonstrated for compact dynamical systems. For our extended version, we shall treat the dynamical system $T: X \rightarrow X$ as a subsystem of a compact metrizable one (Definition 2.1).

For a topological space $X$ to be contained as a subspace in its one point compactification $X \cup\{\infty\}$, and in order for this one point compactification to be metrizable, it is necessary and sufficient that $X$ is a metrizable locally compact separable space.

Definition 2.1 (Subsystem). We say that a (topological) dynamical system $T: X \rightarrow X$ is a (topological) subsystem of $S: Z \rightarrow Z$ when $X \subset Z$ has the induced topology and $T(x)=S(x)$ for every $x \in X$. We also say that $S$ extends $T$ to $Z$. If $T: X \rightarrow X$ and $S: Z \rightarrow Z$ are measurable dynamical systems instead and $X$ is a measurable subset of $Z$, we say that $T$ is a measurable subsystem of $S$.

Lemma 2.2. Suppose $T: X \rightarrow X$ is a measurable subsystem of $S: Z \rightarrow Z$. If $\mathscr{Z}$ is a covering of $Z$ and $\mathscr{C}=X \cap \mathscr{Z}$, then

$$
\mathscr{C}_{T}^{n}=X \cap \mathscr{Z}_{S}^{n} .
$$

Proof. The case $n=1$ is trivial. Assume it is true for $n$. Since $T^{-n}(\mathscr{C})=$ $X \cap S^{-n}(\mathscr{Z})$,

$$
\begin{aligned}
\mathscr{C}_{T}^{n+1} & =\mathscr{C}_{T}^{n} \cap T^{-n}(\mathscr{C}) \\
& =\left(X \cap \mathscr{Z}_{S}^{n}\right) \cap\left(X \cap S^{-n}(\mathscr{Z})\right) \\
& =X \cap \mathscr{Z}_{S}^{n+1} .
\end{aligned}
$$

Lemma 2.3. Whenever $X$ is a topological space with metrizable one point compactification $X^{*}=X \cup\{\infty\}$, any topological dynamical system $T: X \rightarrow X$ is the subsystem of a dynamical system $S: Z \rightarrow Z$, with $Z$ compact metrizable. In this case, the natural projection

$$
\begin{aligned}
\pi: Z & \rightarrow X^{*} \\
x & \mapsto \pi(x)= \begin{cases}x, & x \in X \\
\infty, & x \notin X\end{cases}
\end{aligned}
$$

is continuous.

Proof. Let

$$
Z=\prod_{n=0}^{\infty} X^{*} .
$$

As a denumerable product of compact metrizable spaces, $Z$ is compact metrizable. Identify $X$ with a subset of $Z$ by the injection

$$
\begin{aligned}
\iota: \quad X & \rightarrow Z \\
x & \mapsto\left(T^{n} x\right)_{n=0}^{\infty}
\end{aligned}
$$


If we let $\pi_{n}: Z \rightarrow X^{*}$ be the projection onto the $n$-th coordinate, it is easy to see that $\iota$ is continuous, since $\pi_{n} \circ \iota=T^{n}$ is continuous for every $n \in$ $\mathbb{N}$. Also, restricted to its image, $\iota$ has continuous inverse $\pi_{0}$. Therefore, $X$ is homeomorphic to $\iota(X)$. And with this identification, $T$ is just the restriction to $\iota(X)$ of the shift

$$
S: \quad \begin{array}{rll}
Z & \rightarrow Z \\
\left(x_{n}\right)_{n=0}^{\infty} & \mapsto\left(x_{n+1}\right)_{n=0}^{\infty}
\end{array} .
$$

To see that $\pi$ is continuous at $Z \backslash \iota(X)$, we just need to show that $\pi^{-1}(A)$ is open for every open set $A$ with $\infty \in A$. But since $A=X^{*} \backslash K$ for some compact $K \subset X$, we have that

$$
\pi^{-1}(A)=\pi^{-1}\left(X^{*} \backslash K\right)=Z \backslash \pi^{-1}(K)=Z \backslash \iota(K),
$$

which is open in $Z$, because $\iota(K)$ is compact.

This projection $\pi$, on Lemma 2.3, induces the pseudometric

$$
\widetilde{d}(x, y)=d(\pi(x), \pi(y))
$$

over $Z$. We denote by the same letter $d$ the restriction of this distance to $X$. Since $\pi$ is continuous, this pseudometric is such that the "open balls" are in fact open. Let $X^{c}=Z \backslash X$. Then, these balls are also such that they either contain $X^{c}$, or have empty intersection with it.

Under the conditions of Lemma 2.3 since $X$ is locally compact and separable, it is $\sigma$-compact. That is, $X$ can be written as a denumerable union of compact sets. Since compact sets of $X$ are compact sets of $Z$, it follows that $X \subset Z$ is a Borel set. In this case, the Borel sets of $X$ are Borel sets of $Z$, and we may restrict Borel measures over $Z$ to the Borel sets of $X$. On the other hand, if $\mu$ is a Borel measure over $X$, we can extend it to $Z$ by declaring $\mu(Z \backslash X)=0$. Or, equivalently, we might define $\mu(C)=\mu(C \cap X)$ for every Borel set $C \subset Z$. We shall use the same letter to denote the measure over $Z$ and its restriction to $X$ or any other Borel subset. If we want to make the distinction clear, we may write $\left.\mu\right|_{X}$. According to the following lemma, when $T: X \rightarrow X$ is a subsystem of $S: Z \rightarrow Z$ and $\mu$ is an $S$-invariant finite measure, then $\mu$ is also $T$-invariant.

Lemma 2.4. When $T: X \rightarrow X$ is a measurable subsystem of $S: Z \rightarrow Z$ and $\mu$ is an $S$-invariant finite measure, then $\mu$ is also $T$-invariant.

Proof. Take a measurable set $A \subset X$. Since $\mu$ is $S$-invariant,

$$
\begin{aligned}
\mu(A)+\mu\left(X^{c}\right) & =\mu\left(A \cup X^{c}\right) \\
& =\mu\left(S^{-1}(A) \cup S^{-1}\left(X^{c}\right)\right) \\
& \leq \mu\left(S^{-1}(A) \cup X^{c}\right) \\
& =\mu\left(T^{-1}(A) \cup X^{c}\right) \\
& =\mu\left(T^{-1}(A)\right)+\mu\left(X^{c}\right) .
\end{aligned}
$$

This means that

$$
\mu(A) \leq \mu\left(T^{-1}(A)\right) .
$$

But, on the other hand,

$$
\mu\left(T^{-1}(A)\right) \leq \mu\left(S^{-1}(A)\right)=\mu(A) .
$$


Lemma 2.5. Supose $T: X \rightarrow X$ is a measurable subsystem of $S: Z \rightarrow Z$. Whenever $\mu$ is an $S$-invariant finite measure, we have that for any Borel set $A \subset X$, and any $n \in \mathbb{N}$,

$$
\mu\left(X^{c} \cap S^{-n}(A)\right)=0 .
$$

Proof. Lemma 2.4 implies that $\mu$ is also $T$-invariant. Since $T^{-n}(A)=X \cap$ $S^{-n}(A)$,

$$
\begin{aligned}
\mu\left(X \cap S^{-n}(A)\right) & =\mu\left(T^{-n}(A)\right) \\
& =\mu(A) \\
& =\mu\left(S^{-n}(A)\right) \\
& =\mu\left(X \cap S^{-n}(A)\right)+\mu\left(X^{c} \cap S^{-n}(A)\right) .
\end{aligned}
$$

And therefore, $\mu\left(X^{c} \cap S^{-n}(A)\right)=0$.

\subsection{Kolmogorov-Sinai Entropy}

Consider the finite measure space $(X, \mathscr{B}, \mu)$ and a finite measurable partition $\mathscr{C}$. The partition entropy of $\mathscr{C}$ is

$$
H_{\mu}(\mathscr{C})=\sum_{C \in \mathscr{C}} \mu(C) \log \frac{1}{\mu(C)} .
$$

For the measurable dynamical system $T: X \rightarrow X$, if $\mu$ is a $T$-invariant finite measure, the partition entropy of $T$ with respect to $\mathscr{C}$ is

$$
h_{\mu}(T, \mathscr{C})=\lim _{n \rightarrow \infty} \frac{1}{n} H_{\mu}\left(\mathscr{C}^{n}\right),
$$

and the Kolmogorov-Sinai entropy of $T$ is

$$
h_{\mu}(T)=\sup _{\substack{\mathscr{C} \text { : finite } \\ \text { measurable partition }}} h_{\mu}(T, \mathscr{C}) .
$$

The most basic properties of the partition entropy are a consequence of the concavity of the function

$$
f(x)=-x \log x=x \log \frac{1}{x} .
$$

The following lemma states properties that are standard for probability measures (see [11]). They are easily restated in terms of finite measures, but we shall not need them in this general case.

Lemma 2.6. If $\mu$ is a probability measure and $\mathscr{C}$ and $\mathscr{D}$ finite measurable partitions, then

1. $H_{\mu}(\mathscr{C} \vee \mathscr{D}) \leq H_{\mu}(\mathscr{C})+H_{\mu}(\mathscr{D})$.

2. If $\mu_{n}(C) \rightarrow \mu(C)$ for every $C \in \mathscr{C}$, then $H_{\mu_{n}}(\mathscr{C}) \rightarrow H_{\mu}(\mathscr{C})$.

3. $H_{\mu}(\mathscr{C}) \leq \log N(\mathscr{C})$ 
Lemma 2.7. If $\mu=\alpha \gamma+\beta \nu$ is a convex combination of finite measures and $\mathscr{C}$ is a finite measurable partition, then

$$
\alpha H_{\gamma}(\mathscr{C})+\beta H_{\nu}(\mathscr{C}) \leq H_{\mu}(\mathscr{C}) .
$$

Proof. For each $C \in \mathscr{C}$, the concavity of $x \log \frac{1}{x}$ implies that

$$
\alpha\left(\gamma(C) \log \frac{1}{\gamma(C)}\right)+\beta\left(\nu(C) \log \frac{1}{\nu(C)}\right) \leq \mu(C) \log \frac{1}{\mu(C)} .
$$

Now, one just has to sum up for all $C \in \mathscr{C}$.

Lemma 2.8. If $\mu$ is a finite measure over $X$ with $0 \leq \mu(X) \leq 1$ and $Y \subset X$ is measurable, then

$$
H_{\mu}(\mathscr{C}) \leq H_{\mu}(Y \cap \mathscr{C})+H_{\mu}\left(Y^{c} \cap \mathscr{C}\right)
$$

for any finite measurable partition $\mathscr{C}$.

Proof. Fix $C \in \mathscr{C}$. Let $p=\mu(Y \cap C)$ and $q=\mu\left(Y^{c} \cap C\right)$. If $p=0$, then $\mu(C)=q$, and

$$
\begin{aligned}
\mu(C) \log \frac{1}{\mu(C)} & =q \log \frac{1}{q} \\
& =q \log \frac{1}{q}+p \log \frac{1}{p} .
\end{aligned}
$$

A similar conclusion follows when $q=0$. Assume $p, q \neq 0$. Then, since $p, q \leq$ $\mu(C)$,

$$
\begin{aligned}
\mu(C) \log \frac{1}{\mu(C)} & =p \log \frac{1}{\mu(C)}+q \log \frac{1}{\mu(C)} \\
& \leq p \log \frac{1}{p}+q \log \frac{1}{q} .
\end{aligned}
$$

The lemma follows if we sum up for $C \in \mathscr{C}$.

Notice that our definition of the Kolmogorov-Sinai entropy does not assume that $\mu$ is a probability measure. This will prove to be useful when $X$ is metrizable locally compact (but not necessarily compact). In this case, the set of probability measures is not compact in the weak-* topology, while the set of finite measures with $0 \leq \mu(X) \leq 1$ is indeed compact.

Lemma 2.9. Given a measurable dynamical system $T: X \rightarrow X$ and a finite $T$-invariant measure $\mu$, then, for $\alpha \geq 0$,

$$
h_{\alpha \mu}(T)=\alpha h_{\mu}(T)
$$

Proof. We can assume that $\alpha \neq 0$, since $h_{0}(T)=0$.

For any measurable finite partition $\mathscr{C}$,

$$
\begin{aligned}
\frac{1}{n} \sum_{C \in \mathscr{C}^{n}} \alpha \mu(C) \log \frac{1}{\alpha \mu(C)} & =\frac{\alpha}{n} \sum_{C \in \mathscr{C}^{n}} \mu(C) \log \frac{1}{\alpha \mu(C)} \\
& =\frac{\alpha}{n}\left(\sum_{C \in \mathscr{C}^{n}} \mu(C) \log \frac{1}{\mu(C)}\right)+\frac{\alpha}{n} \mu(X) \log \frac{1}{\alpha}
\end{aligned}
$$

Now, we just have to take the limit for $n \rightarrow \infty$. 
The following lemma will be very important to reduce the prove of the variational principle to the compact case.

Lemma 2.10. Let $S: Z \rightarrow Z$ be a dynamical system and $T: X \rightarrow X$ a subsystem with $X \subset Z$ measurable. If $\mu$ is an $S$-invariant measure, and if

$$
\mathscr{Z}=\left\{Z_{0}, \ldots, Z_{k}\right\}
$$

is a measurable partition of $Z$ such that $Z_{1}, \ldots, Z_{k} \subset X$. Then, $\mu$ is $T$-invariant and

$$
h_{\mu}(S, \mathscr{Z}) \leq h_{\mu}(T, \mathscr{C})
$$

where $\mathscr{C}=X \cap \mathscr{Z}$.

Proof. According to Lemma 2.4, $\mu$ is in fact T-invariant. According to Lemma 2.2 .

$$
\mathscr{C}_{T}^{n}=X \cap \mathscr{Z}_{S}^{n}
$$

Now, Lemma 2.8 implies that

$$
\begin{aligned}
H_{\widetilde{\mu}}\left(\mathscr{Z}_{S}^{n}\right) & \leq H_{\mu}\left(X \cap \mathscr{Z}_{S}^{n}\right)+H_{\mu}\left(X^{c} \cap \mathscr{Z}_{S}^{n}\right) \\
& =H_{\mu}\left(\mathscr{C}_{T}^{n}\right)+H_{\mu}\left(X^{c} \cap \mathscr{Z}_{S}^{n}\right) .
\end{aligned}
$$

Dividing by $n$ and taking the limit as $n \rightarrow \infty$ will prove our claim as soon as we show that the second term on the right side is constant.

Since elements $C \in \mathscr{Z}^{n}$ are of the form

$$
C=C_{0} \cap S^{-1}\left(C_{1}\right) \cap \cdots \cap S^{-n+1}\left(C_{n-1}\right),
$$

with $C_{j} \in \mathscr{Z}$, Lemma 2.5 implies that the only $C \in X^{c} \cap \mathscr{Z}^{n}$ with non null measure is

$$
C=X^{c} \cap \bigcap_{j=0}^{n-1} S^{-1}\left(Z_{0}\right) .
$$

In particular, since $X^{c} \cap \mathscr{Z}$ is a finite partition of $X^{c}$,

$$
\mu(C)=\mu\left(X^{c}\right) .
$$

Therefore,

$$
H_{\mu}\left(X^{c} \cap \mathscr{Z}_{S}^{n}\right)=\mu\left(X^{c}\right) \log \frac{1}{\mu\left(X^{c}\right)}
$$

is constant.

\subsubsection{Conditional Entropy}

Given a probability measure $\mu$ and two finite measurable partitions $\mathscr{C}$ and $\mathscr{D}$, the conditional entropy is an important tool to relate $H_{\mu}(\mathscr{C})$ and $H_{\mu}(\mathscr{D})$. For a measurable set $C$ with $\mu(C)>0$, probability $\mu$ conditioned to $C, \mu(\cdot \mid C)$ is given by

$$
\mu(B \mid C)=\frac{\mu(B \cap C)}{\mu(C)} .
$$

For our purposes, when $\mu(C)=0$, the conditional probability can be defined arbitrarily. 
Definition 2.11 (Conditional Entropy). Given a probability measure $\mu$ and two finite measurable partitions $\mathscr{C}$ and $\mathscr{D}$, the conditional entropy is defined as the expected value

$$
H_{\mu}(\mathscr{D} \mid \mathscr{C})=\sum_{C \in \mathscr{C}} \mu(C) H_{\mu(\cdot \mid C)}(\mathscr{D}) .
$$

Conditional entropy possesses the following properties.

Lemma 2.12. Let $T: X \rightarrow X$ be a measurable dynamical system with $T$-invariant probability measure $\mu$. If $\mathscr{C}$ and $\mathscr{D}$ are two measurable finite partitions, then

$$
h_{\mu}(T, \mathscr{C}) \leq h_{\mu}(T, \mathscr{D})+H_{\mu}(\mathscr{C} \mid \mathscr{D}) .
$$

Proof. This is item (iv) of Theorem 4.12 from [1].

\subsection{Topological Entropy}

A purely topological concept of entropy for a compact system introduced by Adler, Konheim and McAndrew in [1, analogous to the Kolmogorov-Sinai entropy, is the topological entropy. Dinaburg and Goodman proved (see [5, 6]) the variational principle, which states that for metrizable compact systems, the topological entropy is equal to the supremum of the Kolmogorov-Sinai entropies taken over all $T$-invariant probability measures. In [9, Patrão noticed that when the dynamical system admitted a one point compactification, the variational principle still holds as long as we adapt the original definition of topological entropy. In the present paper, we provide a definition of topological entropy which extends the previous definitions and allows us to prove the variational principle for metrizable separable locally compact systems.

Definition 2.13 (Cover Entropy). Given a cover $\mathscr{A}$ of a set $X$, the cover entropy of $\mathscr{A}$ is

$$
H(\mathscr{A})=\log N(\mathscr{A}) .
$$

Motivated by the definition presented in [9], we shall restrict our attention to open covers of a certain type.

Definition 2.14 (Admissible Cover). In a topological space $X$, an open cover $\mathscr{A}$ is said to be admissible when at least one of its elements have compact complement. If every set has compact complement, $\mathscr{A}$ is said to be strongly admissible, or s-admissible for short.

Remark 2.15. If $\tilde{X}$ is a compactification of $X$, then, for any admissible cover $\mathscr{A}$ of $X$, there exists an open cover $\widetilde{\mathscr{A}}$ of $\widetilde{X}$, such that $\mathscr{A}=X \cap \widetilde{\mathscr{A}}$. In fact, we might simply take for $\widetilde{A}$, the family of all open sets $\widetilde{A} \subset \widetilde{X}$ such that $X \cap \widetilde{A} \in \mathscr{A}$. Notice that, $\widetilde{\mathscr{A}}$ is sure to cover $\widetilde{X}$ for the simple fact that there is a compact set $K \subset X$ such that $X \backslash K \in \mathscr{A}$, and therefore $\widetilde{X} \backslash K$ belongs to $\widetilde{\mathscr{A}}$. Since $K$ is covered by elements in $\widetilde{\mathscr{A}}$, we have that $\widetilde{\mathscr{A}}$ is a cover for $\widetilde{X}$.

Definition 2.16 (Topological Entropy). For a dynamical system $T: X \rightarrow X$ and a cover $\mathscr{A}$, the topological entropy of $T$ with respect to $\mathscr{A}$ is

$$
h(T, \mathscr{A})=\lim _{n \rightarrow \infty} \frac{1}{n} H\left(\mathscr{A}^{n}\right) .
$$


The topological entropy of $T$ is

$$
h(T)=\sup _{\mathscr{A}: \text { admissible }} h(T, \mathscr{A}) .
$$

Throughout this paper, the term AKM entropy refers to the original definition of entropy given by Adler, Konheim and McAndrew, while the term topological entropy refers to our modified definition.

Remark 2.17. Notice that as in the case of the AKM entropy and the Kolmogorov-Sinai entropy, the limit in Definition 2.16 exists thanks to the inequality

$$
N(\mathscr{A} \vee \mathscr{B}) \leq N(\mathscr{A}) N(\mathscr{B})
$$

(see Theorem 4.10 in [1]).

We now state some very basic properties satisfied by the topological entropy. Most of the arguments are consequence of the following simple lemma.

Lemma 2.18. Given a dynamical system $T: X \rightarrow X$, let $\mathscr{A}$ and $\mathscr{B}$ be covers of $X$ such that $\mathscr{B} \prec \mathscr{A}$. Then, for all $k \in \mathbb{N}$ and every subset $Y \subset X$,

1. $\mathscr{B}^{k} \prec \mathscr{A}^{k}$.

2. $N_{Y}\left(\mathscr{B}^{k}\right) \leq N_{Y}\left(\mathscr{A}^{k}\right)$.

3. $N_{T^{-1}(Y)}\left(T^{-1}(\mathscr{A})\right) \leq N_{Y}(\mathscr{A})$.

4. $h(T, \mathscr{B}) \leq h(T, \mathscr{A})$.

When the space is compact, it is a simple fact that

$$
h\left(T^{k}\right)=k h(T) .
$$

For the non-compact case, only an inequality follows from a similar argument (see Remark 2.20).

Lemma 2.19. Consider the dynamical system $T: X \rightarrow X$, and let $k \in \mathbb{N}$. Then,

$$
h\left(T^{k}\right) \leq k h(T) .
$$

Proof. Let $\mathscr{A}$ be an admissible cover of $X$. Notice that $\left(\mathscr{A}_{T}^{k}\right)_{T^{k}}^{n}=\mathscr{A}_{T}^{k n}$. So,

$$
k \frac{1}{k n} H\left(\mathscr{A}_{T}^{k n}\right)=\frac{1}{n} H\left(\left(\mathscr{A}_{T}^{k}\right)_{T^{k}}^{n}\right) .
$$

Taking the limit for $n \rightarrow \infty$, we have that

$$
k h(T, \mathscr{A})=h\left(T^{k}, \mathscr{A}_{T}^{k}\right) .
$$

And since $\mathscr{A} \prec \mathscr{A}_{T}^{k}$,

$$
h\left(T^{k}, \mathscr{A}\right) \leq h\left(T^{k}, \mathscr{A}^{k}\right)=k h(T, \mathscr{A}) .
$$

Now, we just have to take the supremum for every admissible cover $\mathscr{A}$ to conclude that

$$
h\left(T^{k}\right) \leq k h(T) .
$$


Remark 2.20. The fact that $\mathscr{A}_{T}^{k}$ is not necessarily an admissible cover obstructed our way into showing that $h\left(T^{k}\right)=k h(T)$. In the case of Kolmogorov-Sinai entropy for instance, the supremum of $h_{\mu}(T, \mathscr{C})$ is taken for every finite measurable partition $\mathscr{C}$. In this case, the demonstration of Lemma 2.19 is easily adapted to show that $h_{\mu}\left(T^{k}\right)=k h_{\mu}(T)$. One just has to notice that since $\mathscr{C}_{T}^{k}$ is a finite measurable partition,

$$
k h_{\mu}(T, \mathscr{C})=h_{\mu}\left(T^{k}, \mathscr{C}_{T}^{k}\right) \leq h_{\mu}\left(T^{k}\right) .
$$

For locally compact separable metrizable systems, the equality $h\left(T^{k}\right)=k h(T)$ will follow from the variational principle (Corollary 3.4).

\subsection{Bowen Entropy}

Bowen introduced in [3] a definition of entropy which coincides with AKM's topological entropy when the dynamical system $T: X \rightarrow X$ is compact metrizable. We shall present Bowen's entropy in a different fashion, easier to compare to the topological entropy.

Choose a distance $d$ in $X$ and, given $\varepsilon>0$, denote by

$$
\mathscr{B}_{d}(\varepsilon)=\left\{B_{d}(x ; \varepsilon) \mid x \in X\right\}
$$

the family of balls of radius $\varepsilon$. Also, for $n \in \mathbb{N}$, we define

$$
d_{n}(x, y)=\max _{0 \leq j<n} d\left(T^{j} x, T^{j} y\right)
$$

In the literature, $(n, \varepsilon)$-spanning sets are usually defined as sets $E \subset X$ for which given any $x \in X$, there exists $y \in E$ such that $d_{n}(x, y) \leq \varepsilon$. We adopt an equivalent definition, but in terms of covers. We do so, in a way that is easier to relate the $(n, \varepsilon)$-spanning sets with our enhanced definition of topological entropy.

Definition 2.21 ((n, 2$)$-Spanning Set). Let $T: X \rightarrow X$ be a dynamical system with a distance $d$. For a given $\varepsilon>0$ and $n \in \mathbb{N}$, a set $E \subset X$ is a $(n, \varepsilon)$-spanning set when

$$
X=\bigcup_{x \in E} B_{d_{n}}(x ; \varepsilon)
$$

That is, the family $\left\{B_{d_{n}}(x ; \varepsilon) \mid x \in E\right\}$ is a cover for $X$.

Definition 2.22 (d-Entropy). Let $T: X \rightarrow X$ be a dynamical system and $d a$ distance for $X$. Given $\varepsilon>0$ and $n \in \mathbb{N}$, define

$$
h^{d}(T, \varepsilon)=\lim _{n \rightarrow \infty} \frac{1}{n} \log N\left(\mathscr{B}_{d_{n}}(\varepsilon)\right)
$$

and

$$
h^{d}(T)=\sup _{\varepsilon>0} h^{d}(T, \varepsilon) .
$$

We denote the usual Bowen entropy by

$$
h_{d}(T)=\sup _{\substack{\varepsilon>0 \\ \text { compact }}} \limsup _{n \rightarrow \infty} \frac{1}{n} \log N_{K}\left(\mathscr{B}_{d_{n}}(\varepsilon)\right) .
$$


Remark 2.23. Again, just like in the case of the topological entropy and the Kolmogorov-Sinai entropy, the limit in Definition 2.22 exists thanks to the inequality

$$
N\left(\mathscr{B}_{d_{n}}(\varepsilon)\right) \leq N\left(\mathscr{B}_{d_{q}}(\varepsilon)\right) N\left(\mathscr{B}_{d_{n-q}}(\varepsilon)\right)
$$

for all $q \in \mathbb{N}$ such that $0<q<n$ (see Theorem 4.10 in [11]).

An alternative way to characterize the $d$-entropy (and Bowen's entropy) is by means of separated sets. Misiurewicz's proof of the variational principle needs this characterization.

Let $T: X \rightarrow X$ be a dynamical system with distance $d$. Given $\varepsilon>0$ and $n \in \mathbb{N}$, we say that a set $S \subset X$ is $(n, \varepsilon)$-separated when for all pairs of distinct points $x, y \in S, d_{n}(x, y) \geq \varepsilon$. For a subset $Y \subset X$ and $\varepsilon>0$, let us write $s(n, \varepsilon, Y)$ for the supremum amongst the cardinalities of $(n, \varepsilon)$-separated subsets of $Y$.

Lemma 2.24. In a dynamical system $T: X \rightarrow X$, where $(X, d)$ is a metric space, given a subset $Y \subset X$ and $\varepsilon>0$, then, for all $n \in \mathbb{N}$,

$$
N_{Y}\left(\mathscr{B}_{d_{n}}(\varepsilon)\right) \leq s(n, \varepsilon, Y) \leq N_{Y}\left(\mathscr{B}_{d_{n}}\left(\frac{\varepsilon}{2}\right)\right) .
$$

Proof. The first inequality follows by the following claim and by the existence (through Zorn's lema) of maximal $(n, \varepsilon)$-separated sets.

Claim. If $E \subset Y$ is a maximal $(n, \varepsilon)$-separated subset of $Y$, then

$$
\mathscr{B}_{E}=\left\{Y \cap B_{d_{n}}(x ; \varepsilon) \mid x \in E\right\}
$$

is a cover of $Y$.

If $\mathscr{B}_{E}$ is not a cover, then, taking $y \in Y \backslash \bigcup_{x \in E} B_{d_{n}}(x ; \varepsilon)$, we have that the set $E \cup\{y\}$ is $(n, \varepsilon)$-separated, infringing the maximality of the set $E$.

For the second inequality, if $S \subset Y$ is a $(n, \varepsilon)$-separated set, and $\mathscr{B} \subset$ $\mathscr{B}_{d_{n}}\left(\frac{\varepsilon}{2}\right)$ covering $Y$, then for each $s \in S$, there exists an $e(s) \in \mathscr{B}$ such that $s \in e(s)$. This mapping is injective. In fact, if $e\left(s_{1}\right)=e\left(s_{2}\right)$, then $d_{n}\left(s_{1}, s_{2}\right)<\varepsilon$. And since $S$ is $(n, \varepsilon)$-separated, we must have $s_{1}=s_{2}$.

The following proposition characterizes the d-entropy in terms of separated sets, and also shows that our formulation of Bowen's entropy (Definition 2.22) is equivalent to that of Bowen himself.

Proposition 2.25. For a dynamical system $T: X \rightarrow X$, where $(X, d)$ is a metric space,

$$
\begin{aligned}
h^{d}(T) & =\sup _{\varepsilon>0} \lim _{n \rightarrow \infty} \frac{1}{n} \log s(n, \varepsilon, X) \\
h_{d}(T)= & \sup _{\substack{\varepsilon>0 \\
K: \text { compact }}} \lim _{n \rightarrow \infty} \frac{1}{n} \log s(n, \varepsilon, K) .
\end{aligned}
$$


Proof. It is immediate from Lemma 2.24, Just take the log, divide by $n$, take the limit for $n \rightarrow \infty$ and the supremum for $\varepsilon>0$ in

$$
N\left(\mathscr{B}_{d_{n}}(\varepsilon)\right) \leq s(n, \varepsilon, X) \leq N\left(\mathscr{B}_{d_{n}}\left(\frac{\varepsilon}{2}\right)\right) .
$$

And for the Bowen entropy, do the same and also take the supremum over compact $K \subset X$ for

$$
N_{K}\left(\mathscr{B}_{d_{n}}(\varepsilon)\right) \leq s(n, \varepsilon, K) \leq N_{K}\left(\mathscr{B}_{d_{n}}\left(\frac{\varepsilon}{2}\right)\right) .
$$

When defining the $d$-entropy, we have used the families $\mathscr{B}_{d_{n}}(\varepsilon)$. Notice that those families are not the same as $\left[\mathscr{B}_{d}(\varepsilon)\right]^{n}$. The following lemma shows that the families $\left[\mathscr{B}_{d}(\varepsilon)\right]^{n}$ would work as well, making the $d$-entropy and the topological entropy much easier to compare.

Lemma 2.26. Let $T: X \rightarrow X$ be a dynamical system with distance $d$. Then,

$$
\begin{aligned}
h^{d}(T) & =\sup _{\varepsilon>0} h\left(T, \mathscr{B}_{d}(\varepsilon)\right) \\
h_{d}(T) & =\sup _{\substack{\varepsilon>0 \\
\text { compact }}} \limsup _{n \rightarrow \infty} \frac{1}{n} \log N_{K}\left(\mathscr{B}_{d}(\varepsilon)^{n}\right) .
\end{aligned}
$$

Proof. It is enough to show that

$$
\left[\mathscr{B}_{d}(\varepsilon)\right]^{n} \prec \mathscr{B}_{d_{n}}(\varepsilon) \prec\left[\mathscr{B}_{d}\left(\frac{\varepsilon}{2}\right)\right]^{n} .
$$

Indeed, this would imply that for any $Y \subset X$,

$$
\frac{1}{n} \log N_{Y}\left(\left[\mathscr{B}_{d}(\varepsilon)\right]^{n}\right) \leq \frac{1}{n} \log N_{Y}\left(\mathscr{B}_{d_{n}}(\varepsilon)\right) \leq \frac{1}{n} \log N_{Y}\left(\left[\mathscr{B}_{d}\left(\frac{\varepsilon}{2}\right)\right]^{n}\right) .
$$

And therefore, the claim for the first equality will follow if we make $n \rightarrow \infty$ and take the supremum for $\varepsilon>0$ and $Y=X$. For the second equality, instead of taking $Y=X$, we also have to take the supremum for every $Y \subset X$ compact.

It is immediate that

$$
\mathscr{B}_{d_{n}}(\varepsilon) \subset\left[\mathscr{B}_{d}(\varepsilon)\right]^{n},
$$

since every ball in the distance $d_{n}$ has the form

$$
B_{d}(x ; \varepsilon) \cap \cdots \cap T^{-(n-1)} B_{d}\left(T^{n-1} x ; \varepsilon\right) .
$$

Therefore, $\left[\mathscr{B}_{d}(\varepsilon)\right]^{n} \prec \mathscr{B}_{d_{n}}(\varepsilon)$. On the other hand, a set $A \in\left[\mathscr{B}_{d}\left(\frac{\varepsilon}{2}\right)\right]^{n}$ has the form

$$
A=B_{d}\left(x_{0} ; \frac{\varepsilon}{2}\right) \cap \cdots \cap T^{-(n-1)} B_{d}\left(x_{n-1} ; \frac{\varepsilon}{2}\right) .
$$

In particular, if $A \neq \emptyset$, by taking $x \in A$, we have that for $j=0, \ldots, n-1$, $d\left(T^{j} x, x_{j}\right)<\frac{\varepsilon}{2}$. So,

$$
B_{d}\left(x_{j} ; \frac{\varepsilon}{2}\right) \subset B_{d}\left(T^{j} x ; \varepsilon\right) .
$$

Therefore,

$$
A \subset B_{d}(x ; \varepsilon) \cap \cdots \cap T^{-(n-1)} B_{d}\left(T^{n-1} x ; \varepsilon\right) \in \mathscr{B}_{d_{n}}(\varepsilon) .
$$


The following lemma states the existence of the Lebesgue number in a form which is easy to apply to the construction of refinements for a given cover.

Lemma 2.27 (Lebesgue Number). Suppose $(X, d)$ is a metric space which admits a compactification $(\widetilde{X}, \widetilde{d})$. Let $\mathscr{A}=X \cap \widetilde{\mathscr{A}}$, where $\widetilde{\mathscr{A}}$ is an open cover of $\tilde{X}$. Then, there exists $\varepsilon>0$ such that

$$
\mathscr{A} \prec \mathscr{B}_{d}(\varepsilon) \text {. }
$$

Proof. Let

$$
C_{\varepsilon}=\left\{x \in \widetilde{X} \mid \exists A \in \widetilde{\mathscr{A}}, B_{\widetilde{d}}(x ; \varepsilon) \subset A\right\}
$$

be the set of all $x \in \widetilde{X}$ such that the balls centered in $x$ with radius $\varepsilon$ is contained in some element of $\widetilde{\mathscr{A}}$. Now, we shall find $\varepsilon>0$ such that $\widetilde{X}=C_{\varepsilon}$.

Claim. $\tilde{X}=\bigcup_{\varepsilon>0} C_{\varepsilon}$.

For each $x \in \widetilde{X}$, there is a $A \in \widetilde{\mathscr{A}}$ containing $x$. This means that there exists $\varepsilon>0$ with $B_{\widetilde{d}}(x ; \varepsilon) \subset A$.

Claim. $C_{\varepsilon} \subset \operatorname{int}\left(C_{\frac{\varepsilon}{2}}\right)$.

For $x \in C_{\varepsilon}$, take $A \in \mathscr{A}$ such that $B_{\widetilde{d}}(x ; \varepsilon) \subset A$. And notice that

$$
B_{\widetilde{d}}\left(x ; \frac{\varepsilon}{2}\right) \subset C_{\frac{\varepsilon}{2}} .
$$

In fact, for $y \in B_{\widetilde{d}}\left(x ; \frac{\varepsilon}{2}\right)$, we have that $B_{\widetilde{d}}\left(y ; \frac{\varepsilon}{2}\right) \subset B_{\widetilde{d}}(x ; \varepsilon)$. That is, $x \in \operatorname{int}\left(C_{\frac{\varepsilon}{2}}\right)$.

Joining both claims, we have that

$$
\widetilde{X}=\bigcup_{\varepsilon>0} \operatorname{int}\left(C_{\varepsilon}\right)
$$

Since $\widetilde{X}$ is compact, there exists $\varepsilon>0$ such that $\widetilde{X}=C_{\varepsilon}$. This means that $\widetilde{\mathscr{A}} \prec \mathscr{B}_{\widetilde{d}}(\varepsilon)$. Taking the intersection with $X$,

$$
\mathscr{A}=X \cap \widetilde{\mathscr{A}} \prec X \cap \mathscr{B}_{\widetilde{d}}(\varepsilon) .
$$

Now, we just have to observe that since

$$
B_{d}(x ; \varepsilon)=X \cap B_{\widetilde{d}}(x ; \varepsilon)
$$

for any $x \in X$,

$$
\mathscr{B}_{d}(\varepsilon) \subset X \cap \mathscr{B}_{\widetilde{d}}(\varepsilon) .
$$

Therefore,

$$
\mathscr{A} \prec \mathscr{B}_{d}(\varepsilon) \text {. }
$$


Finally, we relate the topological entropy and the $d$-entropy.

Proposition 2.28. Let $T: X \rightarrow X$ be a dynamical system and $\underset{\widetilde{X}}{\widetilde{d}}$ a metrizable compactification of $X$. If $d$ is the restriction to $X$ of a distance $\widetilde{d}$ in $\widetilde{X}$, then

$$
h^{d}(T)=\sup _{\widetilde{A}} h(T, X \cap \widetilde{\mathscr{A}}),
$$

where the supremum is taken over all open covers $\widetilde{\mathscr{A}}$ of $\widetilde{X}$.

Proof. The family $\mathscr{D}=\left\{B_{\widetilde{d}}(x ; \varepsilon) \mid x \in X\right\}$ covers $\widetilde{X}$, and is such that $\mathscr{B}_{d}(\varepsilon)=$ $X \cap \mathscr{D}$. This implies that

$$
h^{d}(T) \leq \sup _{\mathscr{\mathscr { A }}: \text { open }} h(T, X \cap \widetilde{\mathscr{A}}) .
$$

Now, Lemma 2.27 implies that for any open cover of $\widetilde{X}, \widetilde{A}$, there exists $\varepsilon>0$ such that

$$
X \cap \widetilde{\mathscr{A}} \prec \mathscr{B}_{d}(\varepsilon) .
$$

And this means that

$$
h(T, X \cap \widetilde{\mathscr{A}}) \leq h\left(T, \mathscr{B}_{d}(\varepsilon)\right) .
$$

Taking the supremum in $\varepsilon$ and applying the Lemma 2.26, we conclude that

$$
h(T, X \cap \widetilde{\mathscr{A}}) \leq h^{d}(T) .
$$

Taking the supremum for all open covers $\widetilde{\mathscr{A}}$,

$$
\sup _{\mathscr{A}: \text { open }} h(T, X \cap \mathscr{\mathscr { A }}) \leq h^{d}(T) \text {. }
$$

When the dynamical system is compact, we know that the $d$-entropy does not depend on the distance $d$. The following corollary to Proposition 2.28 extends this result.

Corollary 2.29. Let $T: X \rightarrow X$ be a dynamical system with $X$ admitting a metrizable compactification $\widetilde{X}$. If $d$ and $c$ are the restriction to $X$ of distancess $\widetilde{d}$ and $\widetilde{c}$ in $\widetilde{X}$, then

$$
h^{d}(T)=h^{c}(T) .
$$

Starting with a technique similar to what we used in the demonstration of Lemma 2.27, we relate Bowen's entropy and the topological entropy.

Proposition 2.30. Let $T: X \rightarrow X$ be a dynamical system with a distance $d$. Then,

$$
h(T) \leq h_{d}(T) \leq h^{d}(T) .
$$


Proof. The Bowen entropy will be calculated according to Lemma 2.26, It is evident from the definitions that $h_{d}(T) \leq h^{d}(T)$.

Given an admissible cover $\mathscr{A}$, take a compact $K \subset X$ such that $K^{c} \in \mathscr{A}$. Let

$$
C_{\varepsilon}=\left\{x \in K \mid \exists A \in \mathscr{A}, B_{d}(x ; 2 \varepsilon) \subset A\right\}
$$

be the set of all $x \in K$ such that the ball centered at $x$ with radius $2 \varepsilon$ is subset of some element in $\mathscr{A}$. Just as in the proof of Lemma 2.27, we can take $\varepsilon>0$ such that $K=C_{\varepsilon}$.

Claim. $\mathscr{A} \prec \mathscr{B}_{d}(\varepsilon)$.

If $B \in \mathscr{B}_{d}(\varepsilon)$ does not intersect $K$, then, $B \subset K^{c} \in \mathscr{A}$. And if $B$ does intersect $K$ then, taking $x \in B \cap K$, from our choice of $\varepsilon$, there is an $A_{x} \in \mathscr{A}$ such that

$$
B \subset B_{d}(x ; 2 \varepsilon) \subset A_{x} .
$$

That is, $\mathscr{A} \prec \mathscr{B}_{d}(\varepsilon)$.

Therefore, defining

$$
\mathscr{D}=\mathscr{B}_{d}(\varepsilon) \cup\left\{K^{c}\right\},
$$

it follows that $\mathscr{A} \prec \mathscr{D}$. Let us partition $X$ in $K_{0}, \ldots, K_{n-1}$ and $\widetilde{K}$, with

$$
\widetilde{K}=K^{c} \cap T^{-1}\left(K^{c}\right) \cap \cdots \cap T^{-(n-1)}\left(K^{c}\right) \in \mathscr{D}^{n}
$$

and, for $m=0, \ldots, n-1$,

$$
K_{m}=K^{c} \cap T^{-1}\left(K^{c}\right) \cap \cdots \cap T^{-(m-1)}\left(K^{c}\right) \cap T^{-m}(K) .
$$

Notice that, in fact, $X=\widetilde{K} \cup \bigcup_{m=0}^{n-1} K_{m}$. So,

$$
N\left(\mathscr{D}^{n}\right) \leq N_{\widetilde{K}}\left(\mathscr{D}^{n}\right)+\sum_{m=0}^{n-1} N_{K_{m}}\left(\mathscr{D}^{n}\right)
$$

Since $\widetilde{K} \in \mathscr{D}^{n}$,

$$
N_{\widetilde{K}}\left(\mathscr{D}^{n}\right)=1 .
$$

For $m=0, \ldots, n-1$, denote by $\mathscr{B}_{m}$ the family of sets of the form

$$
A_{0} \cap T^{-1}\left(A_{1}\right) \cap \cdots \cap T^{-(n-1)}\left(A_{n-1}\right),
$$

where $A_{j}=K^{c}$ for $j=0, \ldots, m-1$, and $A_{j} \in \mathscr{B}_{d}(\varepsilon)$ for $j \geq m$. That is,

$$
\mathscr{B}_{m}=K^{c} \cap T^{-1}\left(K^{c}\right) \cap \cdots \cap T^{-(m-1)}\left(K^{c}\right) \cap T^{-m}\left(\mathscr{B}_{d}(\varepsilon)^{n-m}\right) \subset \mathscr{D}^{n} .
$$

If $\mathscr{F} \subset \mathscr{B}_{d}(\varepsilon)^{n-m}$ is a cover of $K$, then

$$
K^{c} \cap T^{-1}\left(K^{c}\right) \cap \cdots \cap T^{-(m-1)}\left(K^{c}\right) \cap T^{-m}(\mathscr{F}) \subset \mathscr{B}_{m}
$$


is a cover of $K_{m}$. This way,

$$
\begin{aligned}
N_{K_{m}}\left(\mathscr{D}^{n}\right) & \leq N_{K_{m}}\left(\mathscr{B}_{m}\right) \\
& \leq N_{K}\left(\mathscr{B}_{d}(\varepsilon)^{n-m}\right) \\
& \leq N_{K}\left(\mathscr{B}_{d}(\varepsilon)^{n}\right) .
\end{aligned}
$$

And therefore,

$$
\begin{aligned}
N\left(\mathscr{A}^{n}\right) & \leq N\left(\mathscr{D}^{n}\right) \\
& \leq N_{\widetilde{K}}\left(\mathscr{D}^{n}\right)+\sum_{m=0}^{n-1} N_{K_{m}}\left(\mathscr{D}^{n}\right) \\
& \leq 1+\sum_{m=0}^{n-1} N_{K_{m}}\left(\mathscr{D}^{n}\right) \\
& \leq(n+1) N_{K}\left(\mathscr{B}_{d}(\varepsilon)^{n}\right) .
\end{aligned}
$$

Taking the $\log$, dividing by $n$ and making $n \rightarrow \infty$, and using Lemma 2.26, we get that

$$
\begin{aligned}
h(T, \mathscr{A}) & \leq \limsup _{n \rightarrow \infty} \frac{1}{n}\left(\log (n+1)+\log N_{K}\left(\mathscr{B}_{d}(\varepsilon)^{n}\right)\right) \\
& =\limsup _{n \rightarrow \infty} \frac{1}{n} \log N_{K}\left(\mathscr{B}_{d}(\varepsilon)^{n}\right) \\
& \leq h_{d}(T),
\end{aligned}
$$

since $\frac{1}{n} \log (n+1) \rightarrow 0$. Taking the supremum in $\mathscr{A}$,

$$
h(T) \leq h_{d}(T) .
$$

Remark 2.31. When the families $\mathscr{B}_{d}(\varepsilon)$ are admissible covers, Lemma 2.26 warrants that

$$
h_{d}(T) \leq h(T) .
$$

Nonetheless, even when $d$ is the restriction of a distance given in some compactification of the system, $\mathscr{B}_{d}(\varepsilon)$ might not be admissible. As an example, just take $\mathscr{B}_{d}\left(\frac{1}{2}\right)$ in $(0,1)$ with the Euclidean distance. No set in $\mathscr{B}_{d}\left(\frac{1}{2}\right)$ will ever have compact complement and therefore, $\mathscr{B}_{d}\left(\frac{1}{2}\right)$ is not admissible.

\section{Variational Principle}

The preparations made in Section 2 allow us to use Misiurewicz's demonstration of the variational principle almost verbatim. Misiurewicz's original article is 8 . A didatic presentation can be found for example in [11.

We are concerned about the supremum of the Kolmogorov-Sinai entropies over every $T$-invariant Radon probability measure. If there is no such a probability measure, we agree that

$$
\sup _{\mu} h_{\mu}(T)=0 .
$$


According to Lemma 2.9, this is the same as taking the supremum over all $T$-invariant Radon measures $\mu$ with $0 \leq \mu(X) \leq 1$. In this case, there is always an invariant measure. Namely, $\mu=0$.

The following theorem is our main result. The proof will be provided after a few auxiliary results.

Theorem 3.1. Let $T: X \rightarrow X$ be a metrizable locally compact separable dynamical system. Then,

$$
\sup _{\mu} h_{\mu}(T)=h(T)=\min _{d} h_{d}(T) .
$$

The infimum is attained when $d$ is any distance that can be extended to the one point compactification of $X$.

The following lemma is the part of the variational principle that is valid for every topological dynamical system. The lemma remarkably generalizes Proposition 1.4 of 7, since the only hypothesis we impose to the system $T: X \rightarrow X$ is that $T$ is continuous. Notice how the concept of admissible cover allowed us to generalize Proposition 1.4 fo [7] without even appealing to advanced results like the Ergodic Decomposition, the Shannon-McMillan-Breiman or to the Birkhoff Ergodic Theorem.

Lemma 3.2. Let $T: X \rightarrow X$ be a dynamical system and $\mu$ a $T$-invariant Radon probability measure. Then,

$$
h_{\mu}(T) \leq h(T) .
$$

If $X$ is metrizable with distance $d$, then

$$
h_{\mu}(T) \leq h(T) \leq h_{d}(T) .
$$

Proof. The last statement follows from Proposition 2.30. The first part, whose details we will present here, has actually been demonstrated by Misiurewicz. We start by noticing that for every Radon measure, the measure of a set $A$ can be approximated from inside by a compact $K \subset A$.

Let $\mu$ be any $T$-invariant Radon probability measure. We shall show that for any $\mu$-invariant dynamical system $S: X \rightarrow X$,

$$
h_{\mu}(S) \leq h(S)+2+\log 2 .
$$

In particular, this is valid for $T^{n}$ for any $n \in \mathbb{N}$. And then, Lemma 2.19] implies that

$$
\begin{aligned}
h_{\mu}(T) & =\frac{1}{n} h_{\mu}\left(T^{n}\right) \\
& \leq \frac{1}{n} h\left(T^{n}\right)+\frac{2+\log 2}{n} \\
& \leq h(T)+\frac{2+\log 2}{n} \rightarrow h(T) .
\end{aligned}
$$

And this will finish the demonstration.

In order to show the validity of equation (11) for any given $S: X \rightarrow X$, take a finite measurable partition $\mathscr{C}$ such that $h_{\mu}(S) \leq h_{\mu}(S, \mathscr{C})+1$. Choose for each $C \in \mathscr{C}, D_{C} \subset C$ such that

$$
\mu\left(C \backslash D_{C}\right) \leq \frac{1}{N(\mathscr{C}) \log N(\mathscr{C})} .
$$


Let $D^{*}=\bigcup_{C \in \mathscr{C}}\left(C \backslash D_{C}\right)$. Then,

$$
\mu\left(D^{*}\right) \leq \frac{1}{\log N(\mathscr{C})} .
$$

Now, define a finite measurable partition $\mathscr{D}$ and a strongly admissible covering $\mathscr{A}$ by

$$
\begin{aligned}
& \mathscr{D}=\left\{D_{C} \mid C \in \mathscr{C}\right\} \cup\left\{D^{*}\right\} \\
& \mathscr{A}=\left\{D_{C} \cup D^{*} \mid C \in \mathscr{C}\right\} .
\end{aligned}
$$

Claim. $H_{\mu}(\mathscr{C} \mid \mathscr{D}) \leq 1$.

First, notice that for every $C \in \mathscr{C}, \mu\left(C \mid D_{C}\right)=1$. So,

$$
H_{\mu\left(\cdot \mid D_{C}\right)}(\mathscr{C})=0 .
$$

Therefore, using Lemma 2.6.

$$
\begin{aligned}
H_{\mu}(\mathscr{C} \mid \mathscr{D}) & =\mu\left(D^{*}\right) H_{\mu\left(\cdot \mid D^{*}\right)}(\mathscr{C}) \\
& \leq \mu\left(D^{*}\right) \log N(\mathscr{C}) \leq 1 .
\end{aligned}
$$

Claim. $h_{\mu}(S, \mathscr{D}) \leq h(S, \mathscr{A})+\log 2$.

Let us show that

$$
N\left(\mathscr{D}^{n}\right) \leq 2^{n} N\left(\mathscr{A}^{n}\right) .
$$

Taking the logarithm, dividing by $n$ and taking the limit for $n \rightarrow \infty$ gives the claim.

Let $\Lambda \subset\{1, \ldots, k\}^{n}$ with $\# \Lambda=N\left(\mathscr{A}^{n}\right)$, such that

$$
X=\bigcup_{\lambda \in \Lambda}\left(K_{0} \cup K_{\lambda_{0}}\right) \cap \cdots \cap T^{-(n-1)}\left(K_{0} \cup K_{\lambda_{n-1}}\right),
$$

where $\lambda=\left(\lambda_{0}, \ldots, \lambda_{n-1}\right)$. Consider the mapping

$$
f: \Lambda \times\{0,1\}^{n} \rightarrow \mathscr{D}^{n}
$$

that maps $(\lambda, x)$ to $Y_{0} \cap \cdots \cap Y_{n-1}$, where

$$
Y_{j}= \begin{cases}T^{-j} K_{\lambda_{j}}, & x_{j}=1 \\ T^{-j} K_{0}, & x_{j}=0\end{cases}
$$

Since

$$
\begin{aligned}
X & =\bigcup_{\lambda \in \Lambda} T^{0}\left(K_{\lambda_{0}} \cup K_{0}\right) \cap \cdots \cap T^{-(n-1)}\left(K_{\lambda_{n-1}} \cup K_{0}\right) \\
& =\bigcup f\left(\Lambda \times\{0,1\}^{n}\right)
\end{aligned}
$$

and $\mathscr{D}^{n}$ is a partition, we have that the image of $f$ contains every non empty element of $\mathscr{D}^{n}$. 
From the last claims and Lemma 2.12, we have that

$$
\begin{aligned}
h_{\mu}(S) & \leq h_{\mu}(S, \mathscr{C})+1 \\
& \leq h_{\mu}(S, \mathscr{D})+2 \\
& \leq h(S, \mathscr{A})+2+\log 2 \\
& \leq h(S)+2+\log 2,
\end{aligned}
$$

demonstrating equation 1, and concluding the proof.

Remark 3.3. In Lema 3.2 , we have actually shown that

$$
h_{\mu}(T) \leq \sup _{\mathscr{A}: \text { s-admissible }} h(T, \mathscr{A}) \leq h(T) .
$$

Proof of Theorem 3.1. Using Lemma 3.2, we know that

$$
\sup _{\mu} h_{\mu}(T) \leq h(T) \leq \inf _{d} h_{d}(T) \leq \inf _{d} h^{d}(T)
$$

where the infimum is taken over every distance $d$ compatible with the topology in $X$. We just have to show that if $r$ is the restriction to $X$ of some distance in its one point compactification, then,

$$
h^{r}(T) \leq \sup _{\mu} h_{\mu}(T)
$$

In fact, this implies that

$$
h^{r}(T) \leq \sup _{\mu} h_{\mu}(T) \leq h(T) \leq \inf _{d} h_{d}(T) \leq \inf _{d} h^{d}(T) \leq h^{r}(T) .
$$

Using Proposition 2.25, it remains to show that, for each fixed $\varepsilon>0$ and each sequence of $(n, \varepsilon)$-separated sets $E_{n}$, there is a Radon measure $\mu$, which is $T$-invariant and has total measure lower then or equal to 1 , and there is a finite measurable partition $\mathscr{C}$, such that for every $n \in \mathbb{N}$,

$$
\limsup _{n \rightarrow \infty} \frac{1}{n} \log \# E_{n} \leq h_{\mu}(T, \mathscr{C}) \text {. }
$$

We shall take $S: Z \rightarrow Z$ as in Lemma 2.3. find a probability measure $\mu$ over the Borel sets of $Z$ and a partition $\mathscr{Z}$ as in Lemma 2.10, and show that

$$
\limsup _{n \rightarrow \infty} \frac{1}{n} \log \# E_{n} \leq h_{\mu}(S, \mathscr{Z})
$$

Then, Lemma 2.10 will imply the desired conclusion.

The demonstration is very similar to that of Theorem 8.6 of [11, the main difference is that we shall use the pseudometric $\widetilde{r}$ induced by $r$ over $Z$ (see comment after Lemma 2.3). Let us first build up the Radon probability measure $\mu$, and show that it is $S$-invariant. Then, Lemma 2.4 implies that $\mu$ is $T$-invariant. Define

$$
\sigma_{n}=\frac{1}{\# E_{n}} \sum_{x \in E_{n}} \delta_{x}
$$


where $\delta_{x}$ is the Dirac measure with support in $x$. Also define

$$
\mu_{n}=\frac{1}{n} \sum_{j=0}^{n-1} \sigma_{n} \circ S^{-j},
$$

and notice that restricted to $X$,

$$
\mu_{n}=\frac{1}{n} \sum_{j=0}^{n-1} \sigma_{n} \circ T^{-j} .
$$

In fact, since $S^{-j}(Z \backslash X) \subset Z \backslash X$,

$$
\mu_{n}(Z \backslash X)=\frac{1}{n} \sum_{j=0}^{n-1} \sigma_{n}\left(S^{-j}(Z \backslash X)\right)=0 .
$$

Claim. There is a subsequence $n_{k}$ and a Radon probability measure $\mu$ such that $\mu_{n_{k}} \rightarrow \mu$, and such that

$$
\lim _{k \rightarrow \infty} \frac{1}{n_{k}} \log \# E_{n_{k}}=\limsup _{n \rightarrow \infty} \frac{1}{n} \log \# E_{n} .
$$

Also, for any measurable $C \subset Z$ with $\mu(\partial C)=0$,

$$
\lim \mu_{n_{k}}(C)=\mu(C) .
$$

In the weak-* topology, the set of Radon probability measures $\mu$ over $Z$ is easily seen to be closed. The fact that $Z$ is separable implies that the set of Radon measures is separable. The Alaoglu Theorem (Theorem 2.5.2 of [10]) implies that it is compact, and therefore, sequentially compact.

It is clear that there is a subsequence $n_{k}$ such that

$$
\lim _{k \rightarrow \infty} \frac{1}{n_{k}} \log \# E_{n_{k}}=\limsup _{n \rightarrow \infty} \frac{1}{n} \log \# E_{n} .
$$

From the sequential compacity, we can assume that $n_{k}$ is such that $\mu_{n_{k}}$ converges to some $\mu$. The last assertion is a consequence of the Portmanteau Theorem, and can be found in [2], Theorem 2.1, item (v).

Claim. The measure $\mu$ is $S$-invariant.

It is clear that $\mu_{n_{k}} \circ S^{-1} \rightarrow \mu \circ S^{-1}$. In fact, for any continuous $\phi: Z \rightarrow \mathbb{R}$, since $R$ is continuous,

$$
\begin{aligned}
\int \phi \mathrm{d}\left(\mu_{n_{k}} \circ S^{-1}\right) & =\int \phi \circ S \mathrm{~d} \mu_{n_{k}} \\
& \rightarrow \int \phi \circ S \mathrm{~d} \mu \\
& =\int \phi \mathrm{d}\left(\mu \circ S^{-1}\right) .
\end{aligned}
$$


On the other hand,

$$
\begin{aligned}
\left|\int \phi \mathrm{d}\left(\mu_{n_{k}}-\mu_{n_{k}} \circ S^{-1}\right)\right| & =\left|\int \phi \frac{1}{n_{k}} \mathrm{~d}\left(\sigma_{n_{k}}-\sigma_{n_{k}} \circ S^{-n}\right)\right| \\
& =\frac{1}{n_{k}}\left|\int\left(\phi-\phi \circ S^{n_{k}}\right) \mathrm{d} \sigma_{n_{k}}\right| \\
& \leq \frac{1}{n_{k}} \int\left\|\phi-\phi \circ S^{n_{k}}\right\|_{\infty} \mathrm{d} \sigma_{n_{k}} \\
& \leq \frac{1}{n_{k}} \int 2\|\phi\|_{\infty} \mathrm{d} \sigma_{n_{k}} \\
& =\frac{1}{n_{k}} 2\|\phi\|_{\infty} \rightarrow 0 .
\end{aligned}
$$

This implies that

$$
\mu=\lim \mu_{n_{k}}=\lim \mu_{n_{k}} \circ S^{-1}=\mu \circ S^{-1} .
$$

Now, we choose a measurable partition $\mathscr{Z}$. For each $z \in Z$, there exists a ball $B_{z}=B\left(z ; \varepsilon_{z}\right)$ with $\varepsilon_{z}<\frac{\varepsilon}{2}$, such that $\mu\left(\partial B_{z}\right)=0$. Such a $B_{z}$ exists because since the border of such balls are all disjont, there is at most a countable number of reals $\varepsilon>0$ such that $B(z ; \varepsilon)$ has border with non null measure. Since $Z$ is compact and the balls are open, there is a finite number of such balls, $B_{0}, \ldots, B_{n}$ covering $Z$. We can assume that $\left\{B_{0}, \ldots, B_{n}\right\}$ has no proper sub-cover. Let

$$
Z_{j}=B_{j} \backslash\left(B_{1} \cup \cdots \cup B_{j-1}\right) .
$$

Then, $\mathscr{Z}=\left\{Z_{0}, \ldots, Z_{k}\right\}$ is a measurable partition. We can also assume that $Z \backslash X \subset B_{0}=Z_{0}$. That is, $\mathscr{Z}$ satisfies the condidtions of Lemma 2.10.

Claim. For each $C \in \mathscr{Z}^{n}, \mu(\partial C)=0$.

Notice that, since $S$ is continuous, the border operator $\partial$ possesses the following properties.

1. $\partial A=\partial A^{c}$.

2. $\partial\left(A_{1} \cap \cdots \cap A_{k}\right) \subset \partial A_{1} \cup \cdots \cup \partial A_{k}$.

3. $\partial S^{-1}(A) \subset S^{-1}(\partial A)$.

From items (11) and (2), each $Z_{j}=B_{j} \cap B_{1}{ }^{c} \cap \cdots \cap B_{j-1}{ }^{c}$ in $\mathscr{Z}$ has border with null measure. And from items (2) and (3), the same is true for the sets in $\mathscr{Z}^{n}$.

Also, each $C \in \mathscr{Z}^{n}$ has diameter less then $\varepsilon$ in the pseudometric $\widetilde{r}_{n}$, just like in the compact case. 
Claim. $\log \# E_{n}=H_{\sigma_{n}}\left(\mathscr{Z}^{n}\right)$.

Let $C \in \mathscr{Z}^{n}$. If $x, y \in C$, then, there exist $C_{0}, \ldots, C_{n-1} \in \mathscr{Z}$ such that $T^{j} x, T^{j} y \in C_{j}$ for $j=0, \ldots, n-1$. Since each element of $\mathscr{Z}$ has diameter less then $\varepsilon$, we have that $d_{n}(x, y)<\varepsilon$. So, $C$ can contain at most one element of $E_{n}$. That is, $\sigma_{n}(C)=0$ or $\sigma_{n}(C)=\frac{1}{\# E_{n}}$. Therefore,

$$
H_{\sigma_{n}}\left(\mathscr{Z}^{n}\right)=\log \# E_{n} .
$$

Passing from $\sigma_{n}$ to $\mu_{n}$ is the same procedure as in the compact case, as we shall detail right now. Notice that for any measurable finite partition $\mathscr{D}$, Lemma 2.7 implies that

$$
\sum_{j=0}^{n-1} \frac{1}{n} H_{\sigma_{n} \circ S^{-j}}(\mathscr{D}) \leq H_{\mu_{n}}(\mathscr{D}) .
$$

For $n, q \in \mathbb{N}$ with $1<q<n$, take an integer $m$ such that $m q \geq n>m(q-1)$. Then, for every $j=0, \ldots, q-1$,

$$
\begin{aligned}
\mathscr{Z}^{n} & \prec \mathscr{Z}^{j} \vee S^{-j}\left(\mathscr{Z}^{q m}\right) \\
& =\mathscr{Z}^{j} \vee S^{-j}\left(\mathscr{Z}^{q}\right) \vee S^{-(j+q)}\left(\mathscr{Z}^{q}\right) \vee \cdots \vee S^{-(j+(m-1) q)}\left(\mathscr{Z}^{q}\right) .
\end{aligned}
$$

Therefore, using Lemma 2.6.

$$
\begin{aligned}
H_{\sigma_{n}}\left(\mathscr{Z}^{n}\right) & \leq H_{\sigma_{n}}\left(\mathscr{Z}^{j}\right)+H_{\sigma_{n} \circ S^{-(j+0 q)}}\left(\mathscr{Z}^{q}\right)+\cdots+H_{\sigma_{n} \circ S^{-(j+(m-1) q)}}\left(\mathscr{Z}^{q}\right) \\
& \leq H_{\sigma_{n}}\left(\mathscr{Z}^{q}\right)+H_{\sigma_{n} \circ S^{-(j+0 q)}}\left(\mathscr{Z}^{q}\right)+\cdots+H_{\sigma_{n} \circ S^{-(j+(m-1) q)}}\left(\mathscr{Z}^{q}\right) \\
& \leq \log \# \mathscr{Z}^{q}+H_{\sigma_{n} \circ S^{-(j+0 q)}}\left(\mathscr{Z}^{q}\right)+\cdots+H_{\sigma_{n} \circ S^{-(j+(m-1) q)}}\left(\mathscr{Z}^{q}\right) .
\end{aligned}
$$

Summing up in $j=0, \ldots, q-1$,

$$
\begin{aligned}
q \log \# E_{n} & =q H_{\sigma_{n}}\left(\mathscr{Z}^{n}\right) \\
& \leq q \log \# \mathscr{Z}^{q}+\sum_{j=0}^{q-1} \sum_{a=0}^{m-1} H_{\sigma_{n} \circ S^{-(j+a q)}}\left(\mathscr{Z}^{q}\right) \\
& =q \log \# \mathscr{Z}^{q}+\sum_{p=0}^{n-1} H_{\sigma_{n} \circ S^{-p}}\left(\mathscr{Z}^{q}\right)+\sum_{p=n}^{m q-1} H_{\sigma_{n} \circ S^{-p}}\left(\mathscr{Z}^{q}\right) \\
& \leq 2 q \log \# \mathscr{Z}^{q}+n \sum_{p=0}^{n-1} \frac{1}{n} H_{\sigma_{n} \circ S^{-p}}\left(\mathscr{Z}^{q}\right) \\
& \leq 2 q \log \# \mathscr{Z}^{q}+n H_{\mu_{n}}\left(\mathscr{Z}^{q}\right) .
\end{aligned}
$$

Since each element $C \in \mathscr{Z}^{q}$ has border with null measure,

$$
\lim _{k \rightarrow \infty} \mu_{n_{k}}(C)=\mu(C) .
$$


Therefore, dividing by $q n$ and making $k \rightarrow \infty$,

$$
\begin{aligned}
\limsup _{n \rightarrow \infty} \frac{1}{n} \log \# E_{n} & =\lim _{k \rightarrow \infty} \frac{1}{n_{k}} \log \# E_{n_{k}} \\
& \leq 0+\frac{1}{q} \lim _{k \rightarrow \infty} H_{\mu_{n_{k}}}\left(\mathscr{Z}^{q}\right) \\
& =\frac{1}{q} H_{\mu}\left(\mathscr{Z}^{q}\right)
\end{aligned}
$$

for every $q$. Now, one just has to take the limit with $q \rightarrow \infty$ to get

$$
\limsup _{n \rightarrow \infty} \frac{1}{n} \log \# E_{n} \leq h_{\mu}(S, \mathscr{Z}) .
$$

The inequality at Lemma 2.19 was used to demonstrate the variational principle. Now, in its turn, the variational principle allows us to go a bit further.

Corollary 3.4. Consider the metrizable locally compact separable dynamical system $T: X \rightarrow X$, and let $k \in \mathbb{N}$. Then,

$$
h\left(T^{k}\right)=k h(T) .
$$

Proof. Equality holds for the Kolmogorov-Sinai entropy (Remark 2.20). Therefore, the variational principle implies that it is valid for the topological entropy as well.

\section{Application: Lie group endomorphisms}

We finish by considering the entropy of continuous endomorphisms of Lie groups. We extend some results of 4 to endomorphisms which are not necessarily surjective. For a given Lie group $G$, its toral component $T(G)$ is the maximal connected and compact subgroup of the center of $G$. We aim at demonstrating that in certain cases (compact, semisimple, linear reducible and nilpotent), the entropy of an endomorphism of a Lie group $G$ is the entropy of the endomorphism restricted to the toral component $T(G)$.

In order to reduce the general case to the cases covered in [4, a key point is the following lemma, which depends on the variational principle we have developed.

Lemma 4.1. Let $G$ be a connected Lie group and $\phi: G \rightarrow G$ be a continuous endomorphism. There exists a natural number $n$ such that $\phi$ restricted to $H=$ $\phi^{n}(G)$ is surjective. In this case,

$$
h(\phi)=h\left(\left.\phi\right|_{H}\right) .
$$

Proof. For the first claim, we first consider the induced Lie algebra endomorphism $\phi^{\prime}: \mathfrak{g} \rightarrow \mathfrak{g}$ given by the differential of $\phi$ at the identity of $G$. Since $\phi^{\prime}$ is a linear map, there is a natural number $n$ such that $\left(\phi^{\prime}\right)^{n} \mathfrak{g}=\left(\phi^{\prime}\right)^{n+1} \mathfrak{g}$. Putting $H=\phi^{n}(G)$, we have that its Lie algebra is given by $\mathfrak{h}=\left(\phi^{\prime}\right)^{n} \mathfrak{g}$. We have that $\phi(H)=H$, since both are connected subgroups and the Lie algebra of $\phi(H)$ is $\phi^{\prime} \mathfrak{h}=\mathfrak{h}$. Thus $\phi$ restricted to $H$ is surjective. 
For the second claim, notice that, as Lie groups, $H$ and $G$ satisfy the conditions of Theorem 3.1. Also, since $H$ is a countable union of compact subsets, and compact sets of $H$ are also compact sets of $G, H$ is a measurable subset of $G$. Every $\phi$-invariant measure $\mu$ is such that

$$
\begin{aligned}
\mu(H) & =\mu\left(\phi^{-n}(H)\right) \\
& =\mu(G) .
\end{aligned}
$$

Therefore,

$$
\begin{aligned}
h(\phi) & =\sup _{\mu} h_{\mu}(\phi) \\
& =\sup _{\mu} h_{\mu}\left(\left.\phi\right|_{H}\right) \\
& =h\left(\left.\phi\right|_{H}\right) .
\end{aligned}
$$

Using the conclusion and notation of Lemma 4.1, the results in 4 imply that in the cases we are considering,

$$
h(\phi)=h\left(\left.\phi\right|_{H}\right)=h\left(\left.\phi\right|_{T(H)}\right) .
$$

In order to substitute $T(H)$ by $T(G)$, we need to show that in those same cases, $T(H) \subset T(G)$. This is the content of the next lemma.

Lemma 4.2. Let $G$ be a connected reductive or connected nilpotent Lie group and $\phi: G \rightarrow G$ a continuous endomorphism. If $H$ is as in the conclusion of Lemma 4.1, then $T(H) \subset T(G)$.

Proof. Let $\varphi=\phi^{n}$ be as in Lemma 4.1. Then $\varphi$ induces two surjective homomorphisms

$$
\begin{aligned}
\varphi_{1}: \quad G & \rightarrow G / T(G) \\
x & \mapsto \pi(\varphi(x)) \\
\varphi_{2}: H & \rightarrow G / T(G), \\
x & \mapsto \pi(\varphi(x))
\end{aligned}
$$

where $\pi: G \rightarrow G / T(G)$ is the natural projection.

Also, consider the natural projection

$$
\pi_{H}: H \rightarrow \frac{H}{H \cap T(G)}
$$

Since the image of $\varphi_{1}$ and $\varphi_{2}$ are $\frac{H T(G)}{T(G)}$,

$$
\begin{aligned}
G / T(G) & \simeq \frac{H T(G)}{T(G)} \\
& \simeq \frac{H}{H \cap T(G)} \\
& =\pi_{H}(H) .
\end{aligned}
$$


Because $\pi_{H}$ is surjective, it takes the center of $H$ to the center of $\pi_{H}(H)$. In particular, $\pi_{H}(T(H))$ is compact, connected and contained in the center. That is,

$$
\pi_{H}(T(H)) \subset T\left(\pi_{H}(H)\right) .
$$

We claim that $T(H) \subset T(G)$. And this claim follows from equation (2) if we show that $T(\pi(H))=1$. In fact, together with equation (2), this implies that

$$
T(H) \subset \operatorname{ker} \pi_{H}=H \cap T(G) .
$$

In case $G$ is reductive, $G / T(G)$ is semisimple, so is $\pi_{H}(H)$, and semisimple groups have trivial toral component. In case $G$ is nilpotent, Proposition 8 in 4 shows that $G / T(G)$ is simply connected, and so is $\pi_{H}(H)$. Since $\pi_{H}(H)$ is nilpotent and simply connected, $Z\left(\pi_{H}(H)\right)$ is isomorphic to a finite dimensional vector space, and the only compact subgroup of it is the trivial one. Therefore, the toral component of $\pi_{H}(H)$ is trivial.

Proposition 4.3. Let $G$ be a connected and compact Lie group and consider $\phi: G \rightarrow G$ a continuous endomorphism. Then

$$
h(\phi)=h\left(\left.\phi\right|_{T(G)}\right) .
$$

Proof. Consider $H=\phi^{n}(G)$ given by Lemma 4.1. We have that $H$ is a connected and compact Lie group. Using Lemma 4.1 and applying Theorem 6.2 of [4] for $\phi$ restricted to $H$, we get that

$$
h(\phi)=h\left(\left.\phi\right|_{H}\right)=h\left(\left.\phi\right|_{T(H)}\right) .
$$

Now, Lemma 4.2 implies that $T(H) \subset T(G)$, since a compact Lie group is reductive. Therefore, we have that

$$
h(\phi)=h\left(\left.\phi\right|_{T(H)}\right) \leq h\left(\left.\phi\right|_{T(G)}\right) \leq h(\phi) .
$$

In the proof of Proposition 4.3 it was evident that $H=\phi^{n}(G)$ is a compact group. It is also evident that $H$ is nilpotent when $G$ is. But for the semisimple and reductive cases, we need the following lemma.

Lemma 4.4. Let $G$ be a semisimple, or reductive, or nilpotent Lie group. If $\phi: G \rightarrow H$ is a surjective Lie group endomorphism, then $H$ is respectively semisimple, reductive or nilpotent.

Proof. The nilpotent case is trivial. Let $\mathfrak{g}$ denote the Lie algebra of $G$, and $\mathfrak{h}$ be the Lie algebra of $H$.

If $G$ is reductive, then

$$
\mathfrak{g}=\mathfrak{a} \oplus \mathfrak{s},
$$

where $\mathfrak{a}$ is the center of $\mathfrak{g}$, and

$$
\mathfrak{s}=\mathfrak{s}_{1} \oplus \cdots \oplus \mathfrak{s}_{n},
$$

where $\mathfrak{s}_{j}$ are simple ideals of $\mathfrak{g}$. 
Claim. The Lie algebra $\phi^{\prime}(\mathfrak{a})$ is in the center of $\mathfrak{h}$.

Since $\phi^{\prime}$ is surjective,

$$
\left[\mathfrak{g}, \phi^{\prime}(\mathfrak{a})\right]=\left[\phi^{\prime}(\mathfrak{g}), \phi^{\prime}(\mathfrak{a})\right]=\phi^{\prime}([\mathfrak{g}, \mathfrak{a}])=0 .
$$

Claim. The Lie algebra $\phi^{\prime}(\mathfrak{s})$ is semisimple.

Since

$$
\phi^{\prime}(\mathfrak{s})=\phi^{\prime}\left(\mathfrak{s}_{1}\right)+\cdots+\phi^{\prime}\left(\mathfrak{s}_{n}\right),
$$

we just have to show that for each $j=1, \ldots, n, \phi^{\prime}\left(\mathfrak{s}_{j}\right)$ is simple; and for each $j, k=1, \ldots, n$, either $\phi^{\prime}\left(\mathfrak{s}_{j}\right)=\phi^{\prime}\left(\mathfrak{s}_{k}\right)$, or $\phi^{\prime}\left(\mathfrak{s}_{j}\right) \cap \phi^{\prime}\left(\mathfrak{s}_{k}\right)=0$.

Let $I \subset \phi^{\prime}\left(\mathfrak{s}_{j}\right)$ be a non null ideal. Then, $J=\mathfrak{s}_{j} \cap \phi^{\prime-1}(I)$ is a non null ideal of $\mathfrak{s}_{j}$. Since $\mathfrak{s}_{j}$ is simple, $J=\mathfrak{s}_{j}$. That is,

$$
\phi^{\prime}\left(\mathfrak{s}_{j}\right)=\phi^{\prime}(J) \subset I \subset \phi^{\prime}\left(\mathfrak{s}_{j}\right) .
$$

Therefore, $\phi^{\prime}\left(\mathfrak{s}_{j}\right)$ is simple whenever it is non null.

Now, let $I=\phi^{\prime}\left(\mathfrak{s}_{j}\right) \cap \phi^{\prime}\left(\mathfrak{s}_{k}\right)$. Then, $I$ is an ideal of both, $\phi^{\prime}\left(\mathfrak{s}_{j}\right)$ and $\phi^{\prime}\left(\mathfrak{s}_{k}\right)$. Therefore, either $I=0$ or $\phi^{\prime}\left(\mathfrak{s}_{j}\right)=\phi^{\prime}\left(\mathfrak{s}_{k}\right)$.

Denoting by $\mathfrak{h}$ the Lie algebra of $\phi(G)$, we know that

$$
\mathfrak{h}=\phi^{\prime}(\mathfrak{g})=\phi^{\prime}(\mathfrak{a})+\phi^{\prime}(\mathfrak{s})
$$

But, since the intersection of an abelian Lie algebra and a semisimple Lie algebra is 0 , the sum is in fact a direct sum. And this proves that $\phi(G)$ is reductive. If $G$ is semisimple, then $\mathfrak{a}=0$, and therefore, $\mathfrak{h}=\phi^{\prime}(\mathfrak{s})$ is semisimple.

Proposition 4.5. Let $G$ be a connected semisimple Lie group, and let $\phi: G \rightarrow G$ be a continuous endomorphism. Then

$$
h(\phi)=0 .
$$

Proof. Consider $H=\phi^{n}(G)$ given by Lemma 4.1. Then, Lemma 4.4 implies that $H$ is a connected semisimple Lie group, and that

$$
h(\phi)=h\left(\left.\phi\right|_{H}\right)=0,
$$

where the last equality follows from Theorem 5.2 of [4].

Proposition 4.6. Let $G$ be a connected linear reductive or a connected nilpotent Lie group, and let $\phi: G \rightarrow G$ be a continuous endomorphism. Then

$$
h(\phi)=h\left(\left.\phi\right|_{T(G)}\right) .
$$

In particular, any linear transformation $T: V \rightarrow V$ over a finite dimensional vector space $V$ has null topological entropy. 
Proof. Consider $H=\phi^{n}(G)$ given by Lemma 4.1. Then, Lemma 4.4 implies that $H$ is a connected linear reductive or connected nilpotent.

Using Lemma 4.1 and applying Theorem 4.1 (for the nilpotent case) of 4 ] or Corollary 2 (for the linear reductive case) of 4 for $\phi$ restricted to $H$, we get that

$$
h(\phi)=h\left(\left.\phi\right|_{H}\right)=h\left(\left.\phi\right|_{T(H)}\right) .
$$

Now, Lemma 4.2. implies that $T(H) \subset T(G)$, and therefore,

$$
h(\phi)=h\left(\left.\phi\right|_{T(H)}\right) \leq h\left(\left.\phi\right|_{T(G)}\right) \leq h(\phi) .
$$

The last claim follows from the fact that $(V,+)$ is an abelian (a fortiori, nilpotent) Lie group with trivial toral component.

\section{References}

[1] R. Adler, A. Konheim, and M. McAndrew, Topological entropy, Transactions of the American Mathematical Society 114 (1965), no. 2, 309-319.

[2] P. Billingsley, Convergence of probability measures, Wiley Series in Probability and Statistics, Wiley, 1999.

[3] R. Bowen, Entropy for group endomorphisms and homogeneous spaces, Trans. Americ. Math. Soc. 153 (1971), 401-414.

[4] A. Caldas and M. Patrão, Dynamics of endomorphisms of Lie groups, Discrete and Continuous Dynamical Systems 33 (2013), 1351-1363.

[5] E. Dinaburg, The relation between topological entropy and metric entropy, Soviet Math. Dokl. 11 (1969), 13-16.

[6] T. Goodman, Relating topological entropy to measure entropy, Bull. London. Math. Soc. 3 (1971), 176-180.

[7] M. Handel, B. Kitchens, and D. Rudolph, Metrics and entropy for non-compact spaces, Israel Journal of Mathematics 91 (1995), 253-271.

[8] M. Misiurewicz, A short proof of the variational principle for a $\mathbb{Z}_{+}^{\mathbb{N}}$ action on a compact space, Astérisque 40 (1976), 147-157.

[9] M. Patrão, Entropy and its variational principle for non-compact metric spaces, Ergodic Theory and Dynamical Systems 30 (2010), 1529-1542.

[10] G. Pedersen, Analysis now, Graduate Texts in Mathematics, vol. 118, Springer, 1989.

[11] P. Walters, An introduction to ergodic theory, Springer-Verlag, Berlin, 2000. 